\title{
Aktuelle Versorgungskonzepte in der geburtshilflichen Anästhesie
}

\author{
Maximiliaan van Erp · Clemens Ortner · Stefan Jochberger · Klaus Ulrich Klein
}

Eingegangen: 6. Februar 2017 / Angenommen: 4. Juli 2017 / Online publiziert: 25. Juli 2017

(C) Der/die Autor(en) 2017. Dieser Artikel ist eine Open-Access-Publikation.

Zusammenfassung Der folgende Artikel soll vor allem praktisch tätigen Anästhesisten, aber auch Personen anderer Fachdisziplinen, eine Übersicht zu aktuellen Versorgungskonzepten in der geburtshilflichen Anästhesie liefern. Neben einer Zusammenfassung der aus dem Englischen übersetzten „Practice Guidelines for Obstetric Anesthesia 2016“ [1] der American Society of Anesthesiologists geben die Autoren persönliche Anmerkungen zu wichtigen Themen wie Präanästhesie und Vorbereitung, Ausstattung und Personal im Kreißsaal, Durchführung von Allgemeinanästhesie, Periduralanalgesie, Spinalanästhesie, kombinierte Spinal-Epiduralanästhesie, „Single-Shot“-Spinalanästhesie und programmiertes intermittierendes epidurales Boluskonzept.

Schlüsselwörter Geburtshilfliche Anästhesie · Kaiserschnitt · Periduralanalgesie $\cdot$ Leitlinien

\footnotetext{
Dr. med. univ. M. van Erp, MD .

Ass. Prof. PD Dr. C. Ortner, MD .

Assoc. Prof. PD Dr. med. K. U. Klein, MD $(\varangle)$

Universitätsklinik für Anästhesie, Allgemeine

Intensivmedizin und Schmerztherapie, Medizinische

Universität Wien, Währinger Gürtel 18-20, 1090 Wien,

Österreich

ulrich.klein@meduniwien.ac.at

Ass. Prof. PD Dr. C. Ortner, MD

clemens.ortner@meduniwien.ac.at

PD Dr. med. S. Jochberger, MD

Universitätsklinik für Anästhesie und

Intensivmedizin, Medizinische Universität Innsbruck,

Anichstraße 35, 6020 Innsbruck, Österreich

stefan.jochberger@meduniwien.ac.at
}

\section{Recent standards in management of obstetric anesthesia}

Summary The following article contains information not only for the clinical working anaesthesiologist, but also for other specialists involved in obstetric affairs. Besides a synopsis of a German translation of the current "Practice Guidelines for Obstetric Anaesthesia 2016" [1], written by the American Society of Anesthesiologists, the authors provide personal information regarding major topics of obstetric anaesthesia including pre-anaesthesia patient evaluation, equipment and staff at the delivery room, use of general anaesthesia, peridural analgesia, spinal anaesthesia, combined spinal-epidural anaesthesia, single shot spinal anaesthesia, and programmed intermittent epidural bolus.

Keywords Obstetric anaesthesia - Cesarean section • Epidural analgesia $\cdot$ Guidelines

\section{Stellenwert der geburtshilflichen Anästhesie}

Innerhalb der Disziplin „Anästhesiologie und Intensivmedizin“ nimmt die geburtshilfliche Anästhesie und Schmerztherapie eine überaus wichtige, im deutschsprachigen Raum jedoch häufig unterschätzte Rolle ein. Demgegenüber wird der „Obstetric Anesthesia“ in angloamerikanischen Ländern seit Jahrzehnten bedeutender Stellenwert zugewiesen. Aufgrund gesellschaftlicher Veränderungen sowie den Fortschritten in der Reproduktionsmedizin werden zunehmend ältere und teilweise vorerkrankte Frauen schwanger. Wenngleich geburtshilfliche maternale Morbidität und Mortalität weltweit sinken, ist bei zunehmender Anzahl kardiovaskulärer Risikopatientinnen ein relativer Anstieg der indirekten maternalen Morbidität in den Industrieländern zu verzeichnen. Auf jeden 
Fall steigen die Anforderungen an die geburtshilfliche Anästhesie und Schmerztherapie insbesondere in Zentren der Maximalversorgung. Immer häufiger suchen kritisch erkrankte Schwangere anstatt einer Notaufnahme den Kreißsaal auf und profitieren von der kompetenten Versorgung durch einen Anästhesisten als „Intensivmediziner im Kreissaal“. Hierfür sind besondere Kenntnisse im Bereich der Notfallund Intensivmedizin sowie eine gute interdisziplinäre Zusammenarbeit notwendig. Auch eine hohe soziale Kompetenz sowie ein Grundverständnis für die besondere Situation beider Elternteile sind essentiell und erfordern eine große Rücksicht- und Anteilnahme.

\section{Practice Guidelines for Obstetric Anesthesia}

Die folgende Zusammenfassung der „Practice Guidelines for Obstetric Anesthesia“ der American Society of Anesthesiologists (ASA) aus dem Jahr 2016 gibt einen Überblick zur klinischen Evidenz und Expertenmeinung zu zentralen Themen der geburtshilflichen Anästhesie [1]. Die aus dem Englischen übersetzten Richtlinien stellen allgemeine Empfehlungen für die tägliche Arbeit im Kreissaal dar. Die Richtlinien wurden auf Basis einer Kombination aus Literaturrecherche und Expertenmeinung erstellt. Zusätzlich werden im folgenden Artikel von den Autoren persönliche Anmerkungen (als Anmerkung der Autoren gekennzeichnet) zum besseren Verständnis und als weiterführende Information hinzugefügt. Aus Gründen der besseren Lesbarkeit wird auf die gleichzeitige Verwendung männlicher und weiblicher Sprachformen verzichtet.

\section{Anamnese und klinische Untersuchung}

Die aktuelle Studienlage zur Anamnese und klinischen Untersuchung von Gebärenden und Patientinnen zur Schnittentbindung (Sectio) ist dürftig. Klinische Daten belegen, dass beim Vorliegen schwangerschaftsassoziierter Erkrankungen das Risiko für geburtshilfliche und anästhesiologische Komplikationen generell erhöht ist [2-13]. Das Expertenkomitee empfiehlt deshalb eine fokussierte Anamneseerhebung und klinische Untersuchung, wobei Umfang sowie Ausmaß vom individuellen Risiko der Schwangeren abhängen. Zusätzlich wird eine Etablierung beziehungsweise Optimierung spezieller Kommunikationswege zwischen den unterschiedlichen Fachdisziplinen gefordert, um Risiken und Probleme frühzeitig erkennen und gegebenenfalls adäquat handeln zu können.

\section{Empfehlung}

- Fokussierte Anamnese und klinische Untersuchung vor der Durchführung anästhesiologischer Maßnahmen.
- Mütterliche Gesundheit, Schwangerschaftsverlauf, Allergien, Narkosekomplikationen bei Voroperationen, Blutdruckmessung und klinische Untersuchung von Herz, Lunge und Atemweg.

- Bei Planung eines neuroaxialen Narkoseverfahrens Inspektion des Rückens.

- Beim Auftreten spezifischer Probleme ist frühzeitig die Geburtshilfe zu informieren.

- Etablierung eines Kommunikationssystems zur Detektion und Minimierung von Risiken.

Anmerkung der Autoren: Idealerweise sollte jede Patientin, die im Kreißsaal aufgenommen wird, durch einen Anästhesisten gesehen werden. Gerade bei Schwangeren sind sorgfältige Anamnese und klinische Untersuchung besonders wichtig. Neben Fragen nach Allergien haben insbesondere eine sorgfältige Blutungsanamnese sowie die Beurteilung des Atemweges hohen klinischen Stellenwert. Das Vorliegen eines möglicherweise schwierigen Atemweges sollte den Geburtshelfern mitgeteilt und in der Patientenakte dokumentiert werden, da eine Notsectio mit Blitzintubation ein hohes Risikopotential birgt. Prinzipiell ist eine rückenmarksnahe Regionalanästhesie aufgrund eines besseren Nutzen-RisikoVerhältnisses bei Schwangeren vorteilhaft. Gebärende im Kreißsaal sollten auch frühzeitig hinsichtlich ihrer Erwartungshaltung an eine Schmerztherapie evaluiert werden.

Geburtshilfliche Patientinnen mit unauffälliger Allgemeinanamnese benötigen zur Vorbereitung eines geplanten Kaiserschnittes keine internistische Freigabe. Bei der Aufklärung wird das Verfahren erklärt sowie das individuelle Komplikationsrisiko erläutert. Um eine optimale anästhesiologische Betreuung der Schwangeren zu gewährleisten, ist eine enge interdisziplinäre Zusammenarbeit mit Geburtshilfe, Hebammendiensten, Neonatologie, Innere Medizin, Neurologie u. a. eine conditio sine qua non. Idealerweise sollte diese Kooperation in hauseigenen Algorithmen bzw. einem geburtshilflichen „Boardmeeting“ für spezielle Risikopatientinnen eine Berücksichtigung finden.

\section{Thrombozytenzahl}

Die derzeitige Studienlage ist unzureichend, um eine Aussage über die Notwendigkeit einer routinemäßigen Bestimmung der Thrombozytenzahl bei unkompliziertem Schwangerschaftsverlauf treffen $\mathrm{zu}$ können. In einer Studie konnte eine Korrelation zwischen Thrombozytenzahl, Fibrinogenspiegel und der Wahrscheinlichkeit für eine postpartale Blutung (PPH) gezeigt werden [14]. Die Expertenkommission sieht die Indikation zur Thrombozytenzahl-Bestimmung als individuelle Entscheidung, die abhängig von der Anamnese (Blutungsanamnese) und der klinischen Untersuchung getroffen werden muss. 


\section{Empfehlung}

- Von (Blutungs-) Anamnese und klinischer Untersuchung abhängige Entscheidung.

- Bei gesunder Patientin ohne Blutungsrisiko und unauffälligem Schwangerschaftsverlauf nicht unbedingt notwendig.

Anmerkung der Autoren: In der Regel wird im Verlauf der Schwangerschaft ein Blutbild abgenommen und die Thrombozytenzahl bestimmt. Nach Expertenmeinung kann die Anlage einer Spinalanästhesie (SpA) oder Periduralanalgesie (PDA) ab einem Thrombozytenwert zwischen $50.000 / \mathrm{ml}$ und $75.000 / \mathrm{ml}$ von einem erfahrenen Anwender erwogen werden. Entscheidend ist nicht die absolute Thrombozytenzahl, sondern Funktion und Abfall der Thrombozyten über die Zeit sowie klinische Blutungsanamnese und -symptomatik. Die SpA sollte mit einer dünnen Spinalnadel angelegt werden. Unter einem Thrombozyten-Wert von 50.000/ml besteht eine Kontraindikation für die Anlage einer SpA bzw. PDA.

\section{Blutgruppenbestimmung}

Die Literatur vermittelt unzureichende klinische Evidenz, ob eine Blutgruppenbestimmung und Kreuzprobenuntersuchung routinemäßig durchgeführt werden sollten. Es ist nicht notwendig bei einer gesunden Patientin mit unauffälligem Schwangerschaftsverlauf routinemäßig eine Kreuzblutuntersuchung anzufordern. Die Entscheidung, ob Blutgruppe und/oder Kreuzbefund bestimmt werden sollten, sollte individuell erfolgen.

\section{Empfehlung}

- Abhängig von Anamnese und klinischer Untersuchung ist eine Blutgruppenbestimmung und Kreuzblutprobe notwendig durchzuführen.

Anmerkung der Autoren: Im Rahmen der Schwangerschaft sollte eine Blutgruppenbestimmung erfolgen. Die Auskreuzung von Erythrozytenkonzentraten ist definiert und richtet sich nach den Empfehlungen der Querschnittsleitlinien zur Therapie mit Blutkomponenten und Plasmaderivaten. Ein besonderes Blutungsrisiko („Post Partum Haemorrhage“, PPH) zeigen Patientinnen mit Makrosomie, Multipara, fortgeschrittenem maternalem Alter, Zustand nach PPH, erhöhtem „Body Mass Index“, Polyhydramnion, Mehrlingsschwangerschaft, Chorioamnionitis, Frühgeburten, Risiko für Plazenta accreta/increta/percreta, Uterus myomatosus und Zustand nach rezenter Curettage.

\section{CTG-Überwachung}

Klinische Studien zeigen, dass sich die fetalen Herztöne unter neuroaxialen Narkoseverfahren ändern können. Daher empfiehlt das Expertengremium eine
Cardiotokogramm (CTG-) Kontrolle vor und nach der Applikation neuroaxial wirksamer Medikamente [15-22]. Die Durchführung der CTG-Kontrolle muss durch qualifiziertes Personal erfolgen.

\section{Empfehlung}

- Eine CTG-Kontrolle ist vor und nach Gabe neuroaxialer Medikamente empfohlen.

- Eine kontinuierliche CTG-Aufzeichnung ist nicht immer notwendig.

Anmerkung der Autoren: Die simultane Aufzeichnung der fetalen Herztöne und der Wehentätigkeit ist seit Veröffentlichung der Empfehlungen zur Verwendung des fetalen Monitorings von der Federation Internationale de Gynecologie et d'Obstetrique (FIGO) aus dem Jahr 1985 aus der Geburtshilfe nicht mehr weg zu denken. Mit Blick auf eine gute interdisziplinäre Zusammenarbeit ist daher auch für den geburtshilflich tätigen Anästhesisten ein Mindestmaß an Kenntnissen zum CTG und seiner Interpretation (FIGO-Klassifikation) hilfreich [23]. Bei PDA-Anlage in Seitenlage kann das CTG belassen werden. Die Erfassung des CTGs auch während des Transports kann sinnvoll sein, um fetale Herztonänderungen frühzeitig zu erfassen und um entsprechend reagieren zu können (z.B. Not-Sectio), weshalb ein portables System sinnvoll ist.

\section{Aspirationsprophylaxe}

Klare Flüssigkeiten (Wasser, Tee): Die Literatur liefert unzureichende Evidenz bezüglich des Zusammenhanges klarer Flüssigkeiten und dem Reflux- bzw. pulmonalen Aspirationsrisiko während Geburt. Keine Daten sprechen gegen eine moderate Flüssigkeitsaufnahme bei gesunden Gebärenden. Eine Flüssigkeitskarenz bis $2 \mathrm{~h}$ vor Sectio caesarea ist ausreichend.

\section{Empfehlung}

- Bei gesunden Gebärenden spricht nichts gegen eine moderate Flüssigkeitsaufnahme.

- Bei elektiver Sectio ist eine Flüssigkeitskarenz von $2 \mathrm{~h}$ ausreichend.

- Bei Gebärenden mit zusätzlichen Risiken für eine Aspiration oder mit erhöhter Wahrscheinlichkeit einer chirurgischen Intervention sollten längere Karenzzeiten für Flüssigkeiten herangezogen werden.

Feste Nahrung: Es konnte keine spezifische Fastenzeit in der Literatur ermittelt werden, die für maternale Anästhesiekomplikationen prädiktiv ist, daher gilt die allgemeingültige Empfehlung zur perioperativen Nahrungskarenz vor Operationen und Eingriffen. 


\section{Empfehlung}

- Feste Nahrung sollte von Gebärenden vermieden werden.

- Bei geplanten Operationen sollte eine Nüchternheit von $6-8 \mathrm{~h}$ bestehen.

Anmerkung der Autoren: Insbesondere der schwere Reflux mit Regurgitation ist klinisch von Bedeutung und sollte anamnestisch erfragt werden. Im Kreißsaal werden Gebärenden zur oralen Flüssigkeitsaufnahme häufig Wasser, (gezuckerter) Tee, Sport- und Kohlhydratgetränke angeboten. Ein "Preloading“ mit intravenöser Flüssigkeit sollte nur durchgeführt werden, wenn Schwangere beispielsweise aufgrund einer langen Wartezeit bereits Zeichen einer Exsikose zeigen. Vorteilhaft ist ein "Coloading" mit intravenöser Flüssigkeit während der Anlage einer SpA, PDA bzw. vor Allgemeinanästhesie. Eine Opiatzugabe bei PDA kann zu Störungen der Motilität des Magen-Darm-Traktes führen und sollte klinisch berücksichtigt werden.

Antazida, $\mathrm{H}_{2}$-Rezeptor-Antagonisten und Metoclopramid

Klinische Studien konnten zeigen, dass Antazida den $\mathrm{pH}$-Wert des Magens in der peripartalen Phase erhöhen [24-27], erlauben jedoch keine Aussage zur Änderung des Magenvolumens [24, 25]. Eine analoge Feststellung gilt für $\mathrm{H}_{2}$-Rezeptor-Antagonisten [28-30]. Die Metoclopramid-Gabe ist mit einer geringeren Häufigkeit von Übelkeit und Erbrechen vergesellschaftet [31-35]. Die Literatur vermittelt keine Daten über den Zusammenhang von pH-Erhöhung und der Häufigkeit von pulmonaler Aspiration, Übelkeit, Morbidität oder Mortalität von geburtshilflichen Patientinnen nach Aspiration von Mageninhalt.

\section{Empfehlung}

- Vor einer Operation sollte an eine zeitgerechte Gabe von Antazida, $\mathrm{H}_{2}$-Rezeptor-Antagonisten und/oder Metoclopramid zur Aspirationsprophylaxe gedacht werden.

Anmerkung der Autoren: Üblicherweise werden $40 \mathrm{ml}$ Natriumcitrat p. o. unmittelbar vor der Operation sowie Ranitidin $150 \mathrm{mg}$ und/oder $20 \mathrm{mg}$ Paspertin i.v. entsprechend der Pharmakokinetik (z. B. 15-30 min) vor der Operation verabreicht.

\section{Optimaler Zeitpunkt des neuroaxialen Verfahrens}

Metaanalysen randomisiert-kontrollierter Studien zeigen unklare Ergebnisse für spontane, instrumentelle Entbindungen und Sectiones beim Vergleich von frühzeitiger (Muttermund $<4-5 \mathrm{~cm}$ ) und später (Muttermund $>4-5 \mathrm{~cm}$ ) Anlage einer PDA [36-40]. Auch eine Studie, die sich mit dem Vergleich von früher und spä- ter Anlage einer kombinierten Spinal-Periduralanalgesie (CSE) beschäftigte, konnte keine schlüssigen Ergebnisse liefern [41, 42]. Nach Expertenmeinung sollte bereits frühzeitig $(<5 \mathrm{~cm})$ ein neuroaxiales Analgesieverfahren angeboten werden. Die Entscheidung über den Anlagezeitpunkt sollte individuell gemeinsam mit der Gebärenden erfolgen und generell nicht von der Muttermund-Öffnung abhängig gemacht werden.

\section{Empfehlung}

- Der Gebärenden sollte frühzeitig die Möglichkeit eines neuroaxialen Analgesieverfahrens zur Reduktion von Geburtsschmerzen angeboten werden.

- Der Zeitpunkt für eine neuroaxiale Analgesie sollte individuell und unabhängig von der MuttermundÖffnung bestimmt werden.

- Die Gebärende sollte frühzeitig darüber aufgeklärt werden, dass durch ein neuroaxiales Narkoseverfahren die Wahrscheinlichkeit einer Sectio nicht zunimmt.

Anmerkung der Autoren: Der Anästhesist sollte frühzeitig in die geburtshilfliche Schmerztherapie einbezogen werden, wobei ein multidisziplinäres und individuelles Konzept zur Schmerztherapie mit der Gebärenden erarbeitet werden sollte. Die PDA-Anlage sollte ab dem Zeitpunkt des Beginns der Geburt (regelmäßige Wehen) angeboten werden, wobei die PDA über mehrere Tage liegen bleiben kann. Definitiv ist die Indikation zur PDA-Anlage völlig unabhängig von der Weite des Muttermundes (von 0 bis $10 \mathrm{~cm}$ ) und sollte rein aus klinischer Sicht erfolgen. Die Wahrscheinlichkeit einer Sectio nimmt durch die Anlage einer PDA nicht zu. Im Gegenteil, eine PDA geht mit einer Entspannung der Gebärenden und Optimierung des fetalen Sauerstoffangebotes einher und führt deshalb häufig zum Geburtsfortschritt.

\section{Vaginale Entbindung nach vorheriger Sectio}

Die klinische Evidenz erlaubt keine eindeutige Aussage über Entbindungsmodus, Zeitpunkt der Entbindung und über die Auswirkungen eines neuroaxialen Narkoseverfahrens bei Schwangeren, die nach vorhergegangener Sectio caesarea eine vaginale Entbindung wünschten [43-47]. Nach Expertenmeinung sollte die PDA-Anlage frühzeitig erfolgen, um auf die Eventualität einer Sectio vorbereitet zu sein.

\section{Empfehlung}

- Bei Gebärenden, die nach Sectio vaginal entbinden wollen, sollte frühzeitig ein neuroaxiales Analgesieverfahren etabliert werden.

- Die Narkose sollte als Katheter-Verfahren durchgeführt werden, um auf die Eventualität einer Sectio caesarea vorbereitet zu sein. 
Anmerkung der Autoren: Im Falle einer vaginalen Entbindung nach vorheriger Sectio caesarea besteht ein nur minimal erhöhtes Risiko für eine Uterusruptur unter Geburt. Plötzlich einsetzende extreme Schmerzen trotz PDA können ein Hinweis für eine Uterusruptur unter Geburt darstellen.

\section{Spezielle Techniken zur Analgesie und Anästhe- sie}

In der Literatur existieren keine eindeutigen Ergebnisse darüber, ob durch eine frühzeitige Anlage eines neuroaxialen Katheters das maternale oder fetale Langzeitergebnis (Outcome) bei schwieriger Geburt verbessert werden kann. Die Expertenkommission empfiehlt die frühzeitige Anlage eines neuroaxialen Katheters, wenn geburtshilfliche oder anästhesiologische Risiken bestehen, um die Notwendigkeit einer Vollnarkose im Rahmen eines Notfalls zu umgehen.

\section{Empfehlung}

- An die frühzeitige Anlage eines neuroaxialen Katheters sollte bei geburtshilflichen oder anästhesiologischen Risiken gedacht werden, um in einer Notfallsituation auf eine Vollnarkose verzichten zu können.

Anmerkung der Autoren: Eine frühzeitige und großzügige PDA-Anlage erfolgt vor allem bei Nullipara, Multipara, Adipositas, Frühgeburt oder Risikogeburt, da die Geburt häufig erleichtert wird und gegebenfalls leicht ein Aufspritzen der PDA erfolgen kann. Bei kardiovaskulären Risikopatientinnen zur elektiven Sectio kann eine elektive PDA oder low-dose CSE erfolgen, wobei mittels bolusweiser Titration des Lokalanästhetikums langsam ein optimales Anästhesieniveau erreicht wird.

Randomisiert-kontrollierte Studien zeigen eine Reduktion von mütterlichen Schmerzen und Unbehagen beim Einsatz einer PDA (intermittierendes Boluskonzept) im Vergleich zu i. v. Bolusgaben von Opiaten [48, 49]. Die Schmerztherapie mittels PDA ist auch stärker wirksam als der Einsatz von i. m. Opiaten [50]. Es kann jedoch keine klare Aussage zu den Effekten auf die Entbindungszeit und Art der Entbindung getroffen werden. Die Expertenkommission sieht in der PDA eine effektive Methode zur geburtshilflichen Schmerztherapie und empfiehlt die epidurale Applikation von Lokalanästhetika und Opiaten bei Verwendung der PDA.

\section{Empfehlung}

- Die PDA kann als effektive geburtshilfliche Schmerztherapie genutzt werden.

- Die Kombination von epidural applizierten Opiaten und Lokalanästhetika wird empfohlen.
Anmerkung der Autoren: Der Katheter wird mit Naropin 0,125\% (10 ml Naropin 0,2\% plus 7,5 ml $\mathrm{NaCl}$ $0,9 \%)+50 \mu g$ Fentanyl $(1 \mathrm{ml})$ fraktioniert aufgespritzt, alternativ können je nach Erfahrung auch andere Dosierungen vorgenommen werden. Diese Angaben gelten für die normal große und normal gewichtige Schwangere. Bei sehr kleinen Gebärenden muss vorsichtiger aufgespritzt werden und die Menge des Lokalanästhetikums muss entsprechend adaptiert werden. Nach zwanzig Minuten muss der Anästhesist das Ausmaß der sensorischen und motorischen Blockade sowie Neurologie prüfen sowie evtl. bestehende Kreislaufinstabilität und neurologische Symptome erfragen. Die Schwangere sollte bilateral warme und trockene Beine haben als Zeichen der effektiven Sympathikusblockade. Nach einer Wartezeit von 30 min kann mit dem intermittierenden Bolus-Konzept begonnen werden. Es werden 2 Ampullen Naropin 0,2\% (20 ml) mit 2 Ampullen $\mathrm{NaCl}$ 0,9\% (20 ml) plus $100 \mu \mathrm{g}$ Fentanyl (2 ml Ampulle) aufgezogen. Die Pumpe ist so programmiert, dass pro Stunde z. B. ein Bolus von $8 \mathrm{ml}$ gegeben wird. Alternativ kann ein kontinuierlicher Perfusor mit einer Laufrate von $8 \mathrm{ml} / \mathrm{h}$ begonnen werden. Zahlreiche Studien belegen, dass das intermittierende Boluskonzept eine bessere Wirkung bei geringerer muskulärer Blockade zeigt als eine kontinuierliche Gabe mittels Perfusor. Die Gebärende sollte sich immer in Begleitung bewegen und vorher mittels „Kniebeuge“-Versuch in Begleitung prüfen, ob sie genug Kraft in den Beinen hat. Darüber hinaus ist die Gebärende darüber zu informieren, dass die Propriozeption (Gefühl in den Beinen und im Rumpf) durch die PDA verändert sein kann. Eine enge Absprache und Kommunikation mit der Gebärenden und den Hebammendiensten ist notwendig.

Reicht die analgetische Wirkung nicht aus, kann ein zusätzliches Aufspritzen des Katheters zur Wehenschmerzhemmung erfolgen. Dieses wird üblicherweise vorsichtig mit 5-10 ml Naropin 0,1\% vorgenommen, wobei die Wirkung nach 10-20 min zu kontrollieren ist. Es sollte nicht zu einer Störung des Geburtsvorganges mit Beeinträchtigung der Motorik der Beine bzw. Störung der Muskelfunktion während der Austreibephase kommen. Das epidural applizierte Volumen hat in der Regel mehr Einfluss auf die Wirkung als die epidural applizierte Konzentration des Lokalanästhetikums. Im Falle von sehr starken Schmerzen kann auch eine geringe Dosierung (z. B. $25 \mu \mathrm{g}$ ) von Fentanyl hinzugegeben werden. Komplikationen der PDA wie inkomplette Epiduralanalgesie, unilaterale Blockade, lückenhafte Blockade, dislozierter Katheter, Blut im Katheter, intravasale Injektion, Duraperforation, Parästhesien oder radikuläre Schmerzen bei Punktion oder Vorschieben des Katheters, ein subduraler Block, eine totale Spinalanästhesie bis hin zum Kreislaufstillstand sollten allen beteiligten Fachdisziplinen inklusive entsprechender Handlungsalgorithmen bekannt sein.

Eine Metaanalyse randomisiert-kontrollierter Studien zeigte eine bessere analgetische Wirksamkeit bei der Mischung von epidural applizierten Lokalanäs- 
thetika mit Opiaten im Vergleich zur alleinigen Gabe von Lokalanästhetika [51-55]. Es konnten keine signifikanten Daten für die Auswirkung auf Spontanentbindungsrate, Hypotension, Pruritus und 1-Minuten APGAR gezeigt werden [52-63]. Ebenso konnte keine Verbesserung der Analgesie-Qualität beim Vergleich von niedrig dosierten Lokalanästhetika mit Opiaten zu einer reinen hochdosierten LokalanästhetikaApplikation gezeigt werden [64-69]. Die Studien zeigten allerdings eine geringere Rate an motorischen Blockaden beim Einsatz niedrig konzentrierter Lokalanästhetika [64-66, 68-70]. Die Expertenkommission empfiehlt daher den Einsatz von niedrig konzentrierten Lokalanästhetika in Kombination mit Opiaten, um eine möglichst geringe motorische Blockade $\mathrm{zu}$ erreichen.

\section{Empfehlung}

- Niedrig konzentrierte Lokalanästhetika in Kombination mit Opiaten sollten für die neuroaxiale Analgesie hinsichtlich einer möglichst geringen motorischen Blockade angewendet werden.

Anmerkung der Autoren: In einigen Häusern werden sogar Konzentrationen von unter 0,1\% Naropin verwendet, allerdings stellt diese Applikation die Ausnahme dar. Eine hochdosierte Lokalanästhetika-Anwendung sollte nur beim Aufspritzen der PDA zur Sectio mit dem Ziel einer vollen sensorischen und motorischen Blockade erfolgen. Nach Aspirationskontrolle am Katheter erfolgt zunächst eine fraktionierte Gabe von z. B. insgesamt 10(-15) $\mathrm{ml}$ Ropivacain 0,75\% $\pm 25(-50) \mu \mathrm{g}$ Fentanyl. Eine Alternative stellt die fraktionierte Gabe von z. B. insgesamt (15-)20 ml Xylocain $2 \% \pm(25-) 50 \mu \mathrm{g}$ Fentanyl dar. Diese Mischung wirkt theoretisch geringfügig schneller, jedoch in der Regel auch etwas kürzer. Generell ist beim Aufspritzen der PDA immer auf eine Hypotension (in $85 \%$ innerhalb der ersten $10 \mathrm{~min}$ ) zu achten und diese ist frühzeitig $z u$ behandeln. In der klinischen Praxis wird die Gesamtdosis häufig auf drei Bolusgaben verteilt, um gebenfalls eine spinale Applikation aufgrund einer Katheterdislokation zu bemerken und eine hohe Spinalanästhesie zu verhindern.

Eine randomisiert-kontrollierte Studie belegt eine längere Analgesie durch spinal applizierte Opiate im Vergleich zu i. v. verabreichten [71]. Die Literatur vermittelt allerdings keine Evidenz, ob die Kombination von spinalen Opiaten mit Lokalanästhetika von Vorteil für die geburtshilfliche Analgesie ist. Die Expertenkommission stimmt überein, dass die spinale Applikation von Opiaten, mit oder ohne Lokalanästhetika, ein effektives, aber zeitlich limitiertes Mittel zur Schmerztherapie im Rahmen einer vaginalen Geburt darstellt. Es wird auch die Zugabe von Lokalanästhetika empfohlen, um die Wirkdauer zu verlängern und die Analgesiequalität zu verbessern.

\section{Empfehlung}

- Die „Single-Shot“ Applikation intrathekaler Opiate, mit oder ohne Lokalanästhetika, kann als effektive, aber zeitlich limitierte Analgesie für die vaginale $\mathrm{Ge}$ burt genutzt werden.

- Der Einsatz von Lokalanästhetika kann die Wirkdauer verlängern und die Qualität der Analgesie verbessern.

- Wenn davon ausgegangen werden kann, dass die Geburt länger dauern wird oder die Möglichkeit einer operativen Entbindung besteht, sollte allerdings ein Katheterverfahren zur geburtshilflichen Analgesie bevorzugt werden.

Anmerkung der Autoren: Zur alleinigen Therapie des Durchbruchschmerzes ganz am Ende der Geburt kann optional auch eine Spinalanästhesie in geringer Dosierung (engl. „low dose spinal“ oder „Single-Shot-spinal“) mit z. B. Naropin 0,1\% 1,5-2 ml \pm Fentanyl $20 \mu \mathrm{gdurch}$ geführt werden.

Studien konnten zeigen, dass der Einsatz von „Pencil-Point“ Spinalnadeln eine deutliche Reduktion der Inzidenz an postpunktionellem Kopfschmerz bewirkt [72-76]. Die Expertenkommission empfiehlt deshalb die Verwendung, um das Risiko eines postpunktionellen Kopfschmerzes zu minimieren.

\section{Empfehlung}

- Für die Anlage einer Spinalanästhesie soll eine dünne „Pencil-Point“ Spinalnadel verwendet werden, um das Risiko des postpunktionellen Kopfschmerzes zu minimieren.

Anmerkung der Autoren: Üblicherweise wird die Spinalanästhesie mit einer „Pencil-Point“ Spinalnadel $25 \mathrm{G}$ durchgeführt, bei Anamnese eines postspinalen Kopfschmerzes können auch dünnere Nadeln erwogen werden. Im Falle einer Duraperforation während der PDA-Anlage sollte die erneute PDA-Anlage durch einen erfahrenen Anästhesisten durchgeführt werden.

Eine Metaanalyse zeigte ein besseres und schnelleres Anschlagen der Analgesie beim Einsatz einer CSE im Vergleich zu einer reinen PDA [77-83]. Beim Einsatz einer CSE ist die Frequenz motorischer Blockaden allerdings höher [77, 79, 80, 83, 84]. Die Expertenkommission sieht keinen Vorteil für die CSE bei Geburten, die länger als die Wirkung einer Spinalanästhesie andauern könnten. Sollte die Möglichkeit einer chirurgischen Intervention im Rahmen der Geburt bestehen, ist einem Katheterverfahren der Vorzug zu geben.

\section{Empfehlung}

- Wenn die Dauer der Geburt vermutlich länger als die Wirkung der spinalen Medikation oder die Mög- 
lichkeit einer chirurgischen Intervention besteht, sollte ein Katheterverfahren bevorzugt werden.

- Die CSE ermöglicht eine schnell einsetzende und gut wirkende Analgesie.

Anmerkung der Autoren: Per definitionem findet bei der CSE auch immer eine Perforation der Dura statt, was theoretisch zu postspinalem Kopfschmerz führen kann. Allerdings konnte diese Hypothese nicht durch Studien belegt werden.

Eine Metaanalyse zeigte einen geringeren Verbrauch von Analgetika beim Einsatz einer patientengesteuerten PDA („Patient Controlled Epidural Analgesia“, PCEA) im Vergleich zu einer kontinuierlichen PDA bei gleicher analgetischer Wirkung [85-90]. Es konnte ein besseres Ergebnis der PCEA gezeigt werden, wenn eine basale epidurale Hintergrundinfusion mit Lokalanästhetika gegeben wurde [91-95]. In Bezug auf motorische Blockade und Art der Entbindung konnten keine eindeutigen Ergebnisse in der Literatur gefunden werden [91-96]. Ein neuer Therapieansatz ist die PIEB („Programmed Intermittent Epidural Bolus“, PIEB) und wird als Weiterentwicklung der PCEA gesehen. Im Rahmen einer PIEB wird anstelle einer kontinuierlichen Infusionsrate ein vorab definierter Bolus intermittierend appliziert. Durch die intermittierende Bolusgabe konnte die Zahl an motorischen Blockaden deutlich gesenkt werden [97]. Im Rahmen einer Studie wurden 3 unterschiedliche Bolusgrößen und Zeitintervalle $(2,5 \mathrm{ml} / 15 \mathrm{~min}$ vs. $5 \mathrm{ml} / 30 \mathrm{~min}$ vs. $10 \mathrm{ml} / 60 \mathrm{~min}$ ) verglichen. Es zeigte sich, dass bei gleicher Analgesie und Patientenzufriedenheit der größte Bolus mit längstem Zeitintervall (10 ml/60 min) zum geringsten Lokalanästhetikaverbrauch führte [98]. Des Weiteren zeigte eine Untersuchung an 270 Gebärenden [89], dass Gesamtverbrauch an Bupivacain in der Bolus-Gruppe (10 ml Bupivacain 0,1\% mit $2 \mu \mathrm{g} / \mathrm{ml}$ Fentanyl alle 20 min abrufbar) signifikant geringer als nach kontinuierlicher epiduraler Infusion $(10 \mathrm{ml} / \mathrm{h})$ oder PCEA mit Basalrate war. Das Expertengremium sieht in der PCEA eine effektive und flexible Technik zur geburtshilflichen Schmerztherapie und die PCEA sollte einer CSE vorgezogen werden.

\section{Empfehlung}

- Die PIEB bietet eine effektive und flexible Technik zur Behandlung von Wehenschmerzen.

- Die PIEB sollte der kontinuierlichen PDA mittels Perfusor vorgezogen werden, um die applizierten Lokalanästhetikadosen zu reduzieren.

Anmerkung der Autoren: Idealerweise sollte die PIEB (z. B. $8 \mathrm{ml}$ Naropin $0,1 \%+2 \mu \mathrm{g} / \mathrm{ml}$ Fentanyl alle $60 \mathrm{~min}$ ) mit Option einer zusätzlichen Bolusgabe (z. B. $5 \mathrm{ml}$ Naropin 0,1\%+2 $\mu \mathrm{g} / \mathrm{ml}$ Fentanyl, Sperrzeit $10 \mathrm{~min}$, 4 Stundenlimit $60 \mathrm{ml}$ ) angewendet werden, um eine optimale PDA mit erhaltener Sensibilität und Motorik der Beine (,Walking Epidural“) zu ermöglichen.

\section{Räumlichkeiten, Ausrüstung und Personal}

Die Literatur ist unschlüssig bezüglich des Ausrüstungsstandards eines Kreißsaal-Operationsraumes (OP). Das Expertenteam empfiehlt, Ausrüstung, Kreißsaal-OP und Personal mit den gleichen Standards wie einen normalen Operationssaal auszustatten. Des Weiteren sollte Material für die Behandlung von geburtshilflichen und anästhesiologischen Komplikationen vorgehalten werden.

\section{Empfehlung}

- Die Ausrüstung eines Kreißsaal-OPs sollte derjenigen eines normalen OPs entsprechen.

- Spezielle Ausrüstung für geburtshilfliche und anästhesiologische Zwischenfälle sollte vorgehalten werden.

- Auch für die postoperative Betreuung sollten Räumlichkeiten und Personal verfügbar sein.

Anmerkung der Autoren: Bei der Planung moderner Kreißsäle ist darauf zu achten, dass idealerweise neben dem Kreißsaal-OP und Aufwachraum mindestens eine weitere Möglichkeit zur Durchführung einer (Not-) Sectio vorhanden sein sollte (inklusive Monitoring, Beatmung, Linksseitenlagerung). Zudem sollte in allen Kreißsaalzimmern idealerweise ein vernetztes Monitoring, eine Absaugung sowie ein Sauerstoff- und Druckluftanschluss vorhanden sein.

\section{Allgemeinanästhesie, PDA, CSE oder Spinal- anästhesie}

Randomisiert-kontrollierte Studien zeigten bessere 1 und 5 min APGAR-Werte bei PDA im Vergleich zur Intubationsnarkose [99-103] (ITN) und unklare Ergebnisse bei der Betrachtung des umbilicalen pH-Werts [101, 103-105]. Der Vergleich von SpA und ITN konnte keine eindeutigen Ergebnisse bei den 1 und $5 \mathrm{~min}$ APGAR-Werten und des umbilicalen $\mathrm{pH}$-Werts zeigen [100, 106-110]. Der Vergleich von SpA und PDA zeigte unklare Ergebnisse bei Einleitungs-Entbindungs-Zeit, Hypotensionsrate, umbilicalem $\mathrm{pH}$ und APGAR-Wert [100, 111-119]. Der Vergleich von CSE und PDA zeigte ebenfalls unklare Ergebnisse von Hypotensionsrate und APGAR-Werten [101, 103, 120-124]. Auch der Vergleich von CSE und SpA zeigte unschlüssige Ergebnisse in Bezug auf Entbindungszeit, Zeit im OP, Hypotension und APGAR-Werte [125-128]. Laut Expertenkommission sollten für die Entscheidung, welches Narkoseverfahren genutzt werden sollte, die anästhesiologischen, geburtshilflichen und fetalen Risiken, der Patientenwunsch sowie die Einschätzung des Anästhesisten entscheidend sein. Für Sectiones sollte ein neuroaxiales Narkoseverfahren der ITN vorgezogen werden. Bei der Anlage einer SpA sollte unbedingt eine Pencil-Point-Nadel und keine geschliffene Nadel verwendet werden. Bei vorhandener PDA und Indikation 
zur dringenden Sectio sollte der Katheter aufgespritzt werden. Eine ITN ist in manchen Situationen (z. B. fetale Bradykardie, Uterusruptur, massive Hämorrhagie, ausgeprägte frühzeitige Plazentalösung, Nabelschnurvorfall oder Beckenendlage) das Narkoseverfahren der Wahl.

\section{Empfehlung}

- Die Entscheidung über das Narkoseverfahren ist von anästhesiologischen, geburtshilflichen und fetalen Risiken, dem Patientenwunsch und der Einschätzung des Anästhesisten abhängig.

- Bei der Anlage einer SpA sollte eine Pencil-PointNadel verwendet werden.

- Für Sectiones sollte ein neuroaxiales Narkoseverfahren der ITN vorgezogen werden.

- In manchen Situationen (z. B. fetale Bradykardie, Uterusruptur, massive Hämorrhagie, ausgeprägte frühzeitige Plazentalösung, Nabelschnurvorfall oder Beckenendlage) ist die ITN aus Zeitgründen häufig das Anästhesieverfahren der Wahl. Dies trifft vor allem bei drohender Asphyxie des Kindes zu bzw. bei Kontraindikationen zu neuroaxialem Verfahren (schwierige Anatomie, Gerinnungsproblematik).

Anmerkung der Autoren: In gewissen Situationen ist auch ein zweizeitiges Vorgehen anzustreben. Beispielsweise kann bei einer Plazenta accreta/increta/percreta zunächst eine SpA inklusive arteriellem Monitoring angelegt werden und nach Abnabelung des Kindes eine ITN sekundär erfolgen.

\section{I. v. Flüssigkeit}

Randomisiert-kontrollierte Studien zeigen keine eindeutigen Ergebnisse zu den Effekten der Inzidenz einer mütterlichen Hypotonie nach „Preloading/ Coloading“ im Vergleich zu keinem Flüssigkeitsbolus während der SpA-Anlage [129-135]. Die Expertenkommission vermutet allerdings, dass durch „Preloading“ eine Hypotension nach Anlage einer SpA reduziert werden kann, wobei keine Zeit durch diese Maßnahme vergeudet werden sollte.

\section{Empfehlung}

- „Preloading“ und „Coloading“ resultieren wahrscheinlich in weniger Hypotension nach SpA-Anlage.

- Die SpA-Anlage sollte allerdings durch ein „Preloading“ nicht verzögert werden.

Anmerkung der Autoren: Während und unmittelbar nach Anlage der SpA wird intravenös balancierte Flüssigkeit (z. B. 1500-2000 ml Elomel) zur Erhöhung der kardialen Vorlast und Steigerung von Herz-Zeitvolumen und Blutdruck gegeben.

\section{Ephedrin oder Phenylephrin}

Klinische Studien zeigen, dass der Einsatz von i. v. Ephedrin die maternale Hypotensionsrate im Vergleich zu Placebo deutlich senkt [136-140]. Bei einer i.m. Applikation konnte kein eindeutiger Vorteil festgestellt werden [141-143]. Beim Einsatz von Phenylephrin konnte nach Anwendung von hohen Dosen eine Reduktion der Hypotensionsrate gezeigt werden [141, 144-146]. Im direkten Vergleich von Ephedrin und Phenylephrin zeigte sich eine geringere Hypotensionsrate beim Einsatz von i. v. Phenylephrin [147-152]. Die Expertenkommission ist der Meinung, dass beide Substanzen gut zur Therapie der mütterlichen Hypotension nach neuroaxialem Narkoseverfahren genutzt werden können.

\section{Empfehlung}

- Ephedrin und Phenylephrin können zur Therapie einer Hypotension nach SpA-Anlage verwendet werden.

- Bei fehlender maternaler Bradykardie sollte Phenylephrin bevorzugt werden, was zur Verbesserung des umbilicalen arteriellen $\mathrm{pH}$-Wertes führt.

Anmerkung der Autoren: Da die uterinen Gefäße keine Autoregulation zeigen, ist ein adäquater Blutdruck während SpA und PDA essentiell, wobei immer eine Systole von $>100 \mathrm{~mm} \mathrm{Hg}$ (besser Normalwerte) anzustreben ist. In der klinischen Praxis wird häufig ein Vasopressor bereits bei Anstieg der Herzfrequenz bzw. Änderung der Kurve der Pulspletysmographie vor Beendigung der Blutdruckmessung gegeben, um einen Blutdruckabfall zu verhindern. Direkt nach SpA-Anlage kommt es in $85 \%$ zu einer therapiebedürftigen signifikanten Hypotonie, welche in aller Regel gut durch eine kombinierte Flüssigkeit- und Vasopressorgabe (z.B. $100 \mu \mathrm{g}$ Phenylephrin i. $v$. oder $5 \mathrm{mg}$ Ephedrin i. v. als Bolus) beherrschbar ist.

\section{Neuroaxiale Opioide zur postoperativen Schmerz- therapie}

Randomisiert-kontrollierte Studien konnten belegen, dass epidural applizierte Opiate mit einer verbesserten postoperativen Analgesie assoziiert sind als i. v. oder i.m. verabreichte Opiate [153-159]. Allerdings konnte eine geringere Rate an Übelkeit, Erbrechen, Juckreiz und Sedierung als Nebenwirkung für epidurale Opiate nicht gezeigt werden [153-157, 159-164]. Das Expertengremium ist der Meinung, dass epidural applizierte Opiate Vorteile gegenüber parenteral verabreichten Opiaten aufweisen. 


\section{Empfehlung}

- Zur postoperativen Schmerztherapie sollten epidural applizierte Opiate bevorzugt werden.

Anmerkung der Autoren: Nach Sectio caesarea weisen ca. $20 \%$ aller Patientinnen einer numerischen Schmerzscore von $\geq 7$ auf, nach vaginaler Geburt nur etwa $10 \%$ aller Gebärenden. Einen (über 2 Monate) persistierenden Schmerz entwickeln im Durchschnitt 10\% aller geburtshilflichen Patientinnen. Sowohl die intrathekale Applikation von Morphin (z. B. 100-150 $\mathrm{g}$ ) im Rahmen der SpA als auch die epidurale Applikation von Morphin (z. B. 3-4 mg) im Rahmen der PDA reduzieren postpartale Schmerzen innerhalb der ersten 24 h. Ein erhöhter Akutschmerz ist assoziiert mit der Inzidenz der postpartalen Depression und persistierenden Schmerzen, weshalb Schmerzen erstgenommen werden sollten. Deshalb sollten auch erweitere schmerztherapeutische Verfahren (z.B. Morphin-Schmerzpumpe, Musculus transversus abdominis (TAP) Blockade [z.B. $20 \mathrm{ml}$ Naropin 0,375\% pro Seite ultraschallgezielt]) angewendet werden.

\section{Postpartale Tubenligatur}

In der Literaturrecherche konnte kein Vorteil für ein neuroaxiales Narkoseverfahren im Vergleich zu einer ITN gezeigt werden. Ebenso wenig konnte der ideale Zeitpunkt für die Durchführung der Tubenligatur in Bezug auf das mütterliche Outcome festgestellt werden. Das Expertengremium stimmt überein, dass die Patienten 6-8 h vor der geplanten Tubenligatur keine feste Nahrung mehr zu sich nehmen sollten und die Entscheidung über Zeitpunkt und Narkoseform individuell nach vorhandenen Narkoserisiken und Patientenwünschen getroffen werden muss. Die Mitglieder der Expertenkommission bevorzugen mehrheitlich ein neuroaxiales Narkoseverfahren.

\section{Empfehlung}

- Keine feste Nahrung 6-8 h vor Tubenligatur, an eine Aspirationsprophylaxe denken.

- Zeitpunkt und Narkoseform müssen nach vorhandenen Narkoserisiken und Patientenwünschen individuell entschieden werden.

- Neuroaxiale Narkoseverfahren sind der ITN vorzuziehen.

- Die Magenentleerung kann durch während der Geburt gegebene Opiate verzögert sein.

- Das Risiko einer PDA-Dislokation steigt mit der Liegedauer des Katheters.

Anmerkung der Autoren: Im Falle eines Kaiserschnittes mit Tubenligatur kann sich die OP-Dauer verlängern, weshalb häufig eine hoch-normale Dosis bei der SpA appliziert wird.

\section{Postpartale Blutung}

Sowohl die Literatur als auch die ASA-Mitglieder empfehlen die Vorhaltung von Ressourcen zum Management einer postpartalen Blutung [165-172].

\section{Empfehlung}

- Krankenhäuser, die geburtshilfliche Dienste anbieten, müssen eine postpartale Blutung behandeln können.

- Im Notfall muss „0 negativ“ als Bluttransfusion gegeben werden.

- Wenn ein „Cellsaver“ vorhanden ist, soll er auch genutzt werden.

Anmerkung der Autoren: Die postpartale Blutung und damit assoziierte Gefahren werden in der Geburtshilfe häufig unterschätzt, weshalb eine genaue Kenntnis der aktuellen Leitlinien zur Behandlung der postpartalen Blutung essentiell ist. Diese Leitlinien sollten in einem krankenhausspezifischen Algorithmus Berücksichtigung finden und die Behandlung sollte in Form von speziellen Simulationstrainings von den behandelnden Disziplinen (Geburtshilfe, Anästhesie, Hebammendienste, u. a.) geübt werden.

\section{Schwieriger Atemweg}

Studien zeigen, dass das Vorhandensein der Ausrüstung für den schwierigen Atemweg die maternale, fetale und neonatale Komplikationsrate reduziert [173-181]. ASA-Mitglieder sehen es als notwendig an, dass Personal und Ausrüstung für den schwierigen Atemweg vorgehalten wird.

\section{Empfehlungen}

- Die Basisausrüstung für die Atemwegssicherung muss immer verfügbar sein.

- Die Ausrüstung für den schwierigen Atemweg muss mobil und schnell verfügbar sein.

- Ein krankenhausinterner Notfallalgorithmus für den schwierigen Atemweg muss vorhanden sein.

- Bei fehlgeschlagener Intubation muss an eine Larynxmaske oder an Masken-Beutel-Beatmung gedacht werden.

- Wenn eine Ventilation nicht möglich ist, muss unverzüglich ein chirurgischer Atemweg geschaffen werden.

Anmerkung der Autoren: Schwangere gelten spätestens ab der 20. Woche als nicht-nüchtern. Bei Schwangeren am Termin ist das Risiko schwerwiegender Atemwegskomplikationen gegenüber der Normalbevölkerung um das 20-fache erhöht. Das Risiko für einen schwierigen Atemweg beträgt ca. 1:30, das Risiko für eine unmögliche Intubation beträgt ca. 1:300. Neben der Atemwegssicherung bei Einleitung treten die meisten respiratori- 
schen Komplikationen bei der Ausleitung der Narkose auf. In der Geburtshilfe besteht die Besonderheit, dass im Falle eines Not-Kaiserschnittes ein mögliches Problem sehr schnell gelöst wird, um früh möglichst mit der Operation beginnen zu können (z. B. auch Atemwegssicherung durch Larynxmaske).

\section{Herz-Kreislauf-Stillstand}

Die Studienlage ist uneinheitlich für die Evaluierung der Effizienz von Reanimationen in der Geburtshilfe. Es konnte gezeigt werden, dass eine möglichst schnelle Sectio die Situation für die Mutter verbessert. Für das Kind steigt die Überlebenswahrscheinlichkeit ab der 24.-25. Schwangerschaftswoche und einer Sectio binnen $5 \mathrm{~min}$ nach mütterlichem Kreislaufstillstand. Das Expertenkonsortium empfiehlt einen sofortigen Beginn mit Basismaßnahmen (Basic Life Support) und erweiterten Reanimationsmaßnahmen (Advanced Life Support) so schnell als möglich. Während der laufenden Reanimation sollte die Mutter in Linksseitenlage gebracht werden und frühzeitig an eine Sectio („Perimortem Sectio Caesarea“) gedacht werden.

\section{Empfehlung}

- Die Ausrüstung für eine Reanimation sollte sofort verfügbar sein.

- Tritt ein Kreislaufstillstand ein, muss sofort mit der Reanimation begonnen werden.

- Mutter in Linksseitenlage bringen.

- Kann kein Spontankreislauf binnen 4 min hergestellt werden, sollte eine Notsectio erfolgen.

Anmerkung der Autoren: Es besteht eine sehr hohe krankenhausspezifische Variabilität (>7fache Unterschiede) der maternalen Mortalität. In einigen Ländern ist diese aufgrund des zunehmenden maternalen Alters und assoziierter Organdysfunktionen sowie weiterer Risikofaktoren wieder steigend. Zu den besonderen Risikofaktoren zählen zerebrale (z. B. Epilepsie), pulmonale (z. B. Mukoviszidose), kardiale (z. B. Klappenersatz, Herzerkrankung), renale (z.B. Nierenerkrankung), metabolische (z. B. Diabetes, Syndrome) Funktionsstörungen, Zustand nach Organersatz (z. B. Leber-, Nieren-, Lungentransplantation) sowie ein spezieller Habitus (z. B. Wirbelsäulenerkrankungen, Adipositas).

Laut internationalem Konsensus sollte nach 4 min Reanimation ohne Rückkehr eines Spontankreislaufes eine „Perimortem Sectio Caesarea“ unverzüglich durchgeführt werden („Do it on the spot“). Zur Durchführung dieser ist lediglich ein Skalpell und Geburtshelfer notwendig. Um eine kontinuierliche Herzdruckmassage zu ermöglichen, soll die Patientin nicht extra in einen OP transferiert werden.

\section{Literaturempfehlung}

- Wallenborn J: Analgesie und Anästhesie in der Geburtshilfe. Anästh Intensivmed 2017;58:66-84 [97]

- Neuhaus S et al. Why mothers die. Anästhesist 2016;65:281-94 [182]

- Difficult Airway Society: Master Algorithm Obstetric Anesthesia 2016 [183]

- ASA: Practice Guidelines for Obstetric Anesthesia 2016 [1]

- ÖGARI: Regionalanästhesie unter gerinnungshemmender Medikation 2016 [184]

- AWMF Leitlinie: Hygieneempfehlungen für die Regionalanästhesie 2014 [185]

- AWMF Leitlinie: Peripartale Blutungen, Diagnostik und Therapie 2016 [186]

- AWMF Leitlinie: Therapie hypertensiver Schwangerschaftserkrankungen 2013 [187]

- AWMF Leitlinie: Anwendung des CTG während Geburt 2013 [188]

- AWMF Leitlinie: Frühgeborene an der Grenze zur Lebensfähigkeit 2014 [189]

- Zur Frage der postoperativen Überwachung von Kaiserschnittpatientinnnen. Anästh Intensiv 2016; 57:47-50 [190]

- Einsatz von Lachgas zur Schmerztherapie unter der Geburt. Anästh Intensiv 2014;55:679-682 [191]

Acknowledgements Open access funding provided by Medical University of Vienna.

Interessenkonflikt M. Van Erp, C. Ortner, C. Jochberger und K. U. Klein geben an, dass kein Interessenkonflikt besteht.

Open Access Dieser Artikel wird unter der Creative Commons Namensnennung 4.0 International Lizenz (http:// creativecommons.org/licenses/by/4.0/deed.de) veröffentlicht, welche die Nutzung, Vervielfältigung, Bearbeitung, Verbreitung und Wiedergabe in jeglichem Medium und Format erlaubt, sofern Sie den/die ursprünglichen Autor(en) und die Quelle ordnungsgemäß nennen, einen Link zur Creative Commons Lizenz beifügen und angeben, ob Änderungen vorgenommen wurden.

\section{Literatur}

1. Practice guidelines for obstetric anesthesia: an updated reportbytheAmericanSocietyofAnesthesiologistsTaskForce on Obstetric Anesthesia and the Society for Obstetric Anesthesia and Perinatology. Anesthesiology 2016;124:270-300. doi:10.1097/ALN.0000000000000935.

2. Aya AG, Vialles N, Tanoubi I, Mangin R, Ferrer JM, Robert C, Ripart J, de La Coussaye JE. Spinal anesthesia-induced hypotension: a risk comparison between patients with severe preeclampsia and healthy women undergoing preterm cesarean delivery. Anesth Analg. 2005;101:869-75, table of contents.

3. Bateman BT, Bansil P, Hernandez-Diaz S, Mhyre JM, Callaghan WM, Kuklina EV. Prevalence, trends, and outcomes of chronic hypertension: a nationwide sample of delivery admissions. Am J Obstet Gynecol. 2012;206(2):134.e1-134.e8. 
4. Crosby ET. Obstetrical anaesthesia for patients with the syndrome of haemolysis, elevated liver enzymes and low platelets. Can JAnaesth. 1991;38:227-33.

5. Goodall PT, Ahn JT, Chapa JB, Hibbard JU. Obesity as a risk factor for failed trial of labor in patients with previous cesarean delivery. Am J Obstet Gynecol. 2005;192:1423-6.

6. Grotegut CA, Kuklina EV, Anstrom KJ, Heine RP, Callaghan WM, Myers ER, James AH. Factors associated with the change in prevalence of cardiomyopathy at delivery in the period 2000-2009: a population-based prevalence study. BJOG. 2014;121:1386-94.

7. Leffert LR, Clancy CR, Bateman BT, Bryant AS, Kuklina EV. Hypertensive disorders and pregnancy-related stroke: frequency, trends, risk factors, and outcomes. Obstet Gynecol. 2015;125:124-31.

8. Mhyre JM, Bateman BT, Leffert LR. Influence of patient comorbidities on the risk of near-miss maternal morbidity or mortality. Anesthesiology. 2011;115:963-72.

9. Naef RW 3rd, Chauhan SP, Chevalier SP, Roberts WE, Meydrech EF, Morrison JC. Prediction of hemorrhage at cesarean delivery. Obstet Gynecol. 1994;83:923-6.

10. Robinson HE, O'Connell CM, Joseph KS, McLeod NL. Maternal outcomes in pregnancies complicated by obesity. Obstet Gynecol. 2005;106:1357-64.

11. Suelto MD, Vincent RD Jr., Larmon JE, Norman PF, Werhan CF. Spinal anesthesia for postpartum tubal ligation after pregnancy complicated by preeclampsia or gestational hypertension. Reg Anesth Pain Med. 2000;25:170-3.

12. von Ungern-Sternberg BS, Regli A, Bucher E, Reber A, Schneider MC. Impact of spinal anaesthesia and obesity on maternal respiratory function during elective caesarean section. Anaesthesia. 2004;59:743-9.

13. Weiner MM, Vahl TP, Kahn RA. Case scenario: cesarean section complicated by rheumatic mitral stenosis. Anesthesiology. 2011;114:949-57.

14. Simon L, Santi TM, Sacquin P, Hamza J. Pre-anaesthetic assessment of coagulation abnormalities in obstetric patients: usefulness, timing and clinical implications. $\mathrm{Br} \mathrm{J}$ Anaesth. 1997;78:678-83.

15. AbboudTK, KhooSS, Miller F, DoanT, Henriksen EH. Maternal, fetal, and neonatal responses after epidural anesthesia with bupivacaine, 2-chloroprocaine, or lidocaine. Anesth Analg. 1982;61:638-44.

16. Abouleish E. Foetal bradycardia during caudal analgesia: a discussion of possible causative factors. Br J Anaesth. 1976;48:481-4.

17. Boehm FH, Woodruff LF Jr., Growdon JH Jr.. The effect of lumbar epidural anesthesia on fetal heart rate baseline variability. Anesth Analg. 1975;54:779-82.

18. Jouppila P, Jouppila R, Kaar K, Merila M. Fetal heart rate patterns and uterine activity after segmental epidural analgesia. Br J Obstet Gynaecol. 1977;84:481-6.

19. Spencer JA, Koutsoukis M, Lee A. Fetal heart rate and neonatal condition related to epidural analgesia in women reaching the second stage of labour. Eur J Obstet Gynecol Reprod Biol. 1991;41:173-8.

20. Swayze CR, Skerman JH, Walker EB, Sholte FG. Efficacy of subarachnoid meperidine for labor analgesia. Reg Anesth. 1991;16:309-13.

21. Stavrou C, Hofmeyr GJ, Boezaart AP. Prolonged fetal bradycardia during epidural analgesia. Incidence, timing and significance. SAfr Med J. 1990;77:66-8.

22. Zilianti M, Salazar JR, Aller J, Aguero O. Fetal heart rate and $\mathrm{pH}$ of fetal capillary blood during epidural analgesia in labor. Obstet Gynecol. 1970;36:881-6.

23. Ayres-de-Campos D, Spong CY, Chandraharan E, Panel FIFMEC. FIGO consensus guidelines on intrapartum fe- tal monitoring: cardiotocography. Int J Gynaecol Obstet. 2015;131:13-24.

24. Dewan DM, Floyd HM, Thistlewood JM, Bogard TD, Spielman FJ. Sodium citrate pretreatment in elective cesarean section patients. Anesth Analg. 1985;64:34-7.

25. Jasson J,LefevreG, TalletF, Talafre ML, LegagneuxF, Conseiller C. Oral administration of sodium citrate before general anesthesia in elective cesarean section. Effect on pH and gastric volume. Ann Fr Anesth Reanim. 1989;8:12-8.

26. Ormezzano X, Francois TP, Viaud JY, Bukowski JG, Bourgeonneau MC, Cottron D, Ganansia MF, Gregoire FM, Grinand MR, Wessel PE. Aspiration pneumonitis prophylaxis in obstetric anaesthesia: comparison of effervescent cimetidine-sodium citrate mixture and sodium citrate. Br J Anaesth. 1990;64:503-6.

27. Wig J, Biswas GC, Malhotra SK, Gupta AN. Comparison of sodium citrate with magnesium trisilicate as pre-anaesthetic antacid in emergency caesarean sections. Indian J Med Res. 1987;85:306-10.

28. Lin CJ, Huang CL, Hsu HW, Chen TL. Prophylaxis against acid aspiration in regional anesthesia for elective cesarean section: a comparison between oral single-dose ranitidine, famotidineand omeprazole assessed with fiberoptic gastric aspiration. Acta Anaesthesiol Sin. 1996;34:179-84.

29. O'Sullivan G, Sear JW, Bullingham RE, Carrie LE. The effect of magnesium trisilicate mixture, metoclopramide and ranitidine on gastric $\mathrm{pH}$, volume and serum gastrin. Anaesthesia. 1985;40:246-53.

30. Qvist N, Storm K, HolmskovA. Cimetidine as pre-anesthetic agent for cesarean section: perinatal effects on the infant, the placental transfer of cimetidine and its elimination in the infants. J Perinat Med. 1985;13:179-83.

31. Cooke RD, Comyn DJ, Ball RW. Prevention of postoperative nausea and vomiting by domperidone: a double-blind randomized study using domperidone, metoclopramide and a placebo. SAfr Med J. 1979;56:827-9.

32. Danzer BI, Birnbach DJ, Stein DJ, Kuroda MM, Thys DM. Does metoclopramide supplement postoperative analgesia using patient-controlled analgesia with morphine in patients undergoingelective cesarean delivery? RegAnesth. 1997;22:424-7.

33. Lussos SA, Bader AM, Thornhill ML, Datta S. The antiemetic efficacy and safety of prophylactic metoclopramide for elective cesarean delivery during spinal anesthesia. Reg Anesth. 1992;17:126-30.

34. Pan PH, Moore CH. Comparing the efficacy of prophylactic metoclopramide, ondansetron, and placebo in cesarean section patients given epidural anesthesia. J Clin Anesth. 2001;13:430-5.

35. Stein DJ, Birnbach DJ, Danzer BI, Kuroda MM, Grunebaum A, Thys DM. Acupressure versus intravenous metoclopramide to prevent nausea and vomiting during spinal anesthesia for cesarean section. Anesth Analg. 1997;84:342-5.

36. Chestnut DH, McGrath JM, Vincent RD Jr., Penning DH, Choi WW, Bates JN, McFarlane C. Does early administration of epidural analgesia affect obstetric outcome in nulliparous women who are in spontaneous labor? Anesthesiology. 1994;80:1201-8.

37. Chestnut DH, Vincent RD Jr., McGrath JM, Choi WW, Bates JN. Does early administration of epidural analgesia affect obstetric outcome in nulliparous women who are receiving intravenous oxytocin? Anesthesiology. 1994;80:1193-200.

38. Luxman D, Wolman I, Groutz A, Cohen JR, Lottan M, Pauzner D, David MP. The effect of early epidural block administration on the progression and outcome of labor. IntJObstetAnesth. 1998;7:161-4. 
39. Ohel G, Gonen R, Vaida S, Barak S, Gaitini L. Early versus late initiation of epidural analgesia in labor: Does it increase the risk of cesarean section? A randomized trial. Am J Obstet Gynecol. 2006;194:600-5.

40. Wang F, ShenX, GuoX, Peng Y, GuX, Labor Analgesia Examining Group. Epidural analgesia in the latent phase of labor and the risk of cesarean delivery: a five-year randomized controlled trial. Anesthesiology. 2009;111(4):871-80.

41. Wang LZ, Chang XY, Hu XX, Tang BL, Xia F. The effect on maternal temperature of delaying initiation of the epidural component of combined spinal-epidural analgesia for labor: a pilot study. IntJ Obstet Anesth. 2011;20:312-7.

42. Wong CA, McCarthy RJ, Sullivan JT, Scavone BM, Gerber SE, Yaghmour EA. Early compared with late neuraxial analgesia in nulliparous labor induction: a randomized controlled trial. Obstet Gynecol. 2009;113:1066-74.

43. Carlsson C, Nybell-Lindahl G, Ingemarsson I. Extradural blockin patients who have previouslyundergone caesarean section. BrJ Anaesth. 1980;52:827-30.

44. Flamm BL, Lim OW, Jones C, Fallon D, Newman LA, Mantis JK. Vaginal birth after cesarean section: results of a multicenter study. Am J Obstet Gynecol. 1988;158:1079-84.

45. Meehan FP, Burke G, Kehoe JT. Update on delivery following prior cesarean section: a 15-year review 1972-1987. Int J GynaecolObstet. 1989;30:205-12.

46. Sakala EP, Kaye S, Murray RD, Munson LJ. Epidural analgesia. Effect on the likelihood of a successful trial of labor after cesarean section. J Reprod Med. 1990;35:886-90.

47. Stovall TG, Shaver DC, Solomon SK, Anderson GD. Trial of labor in previous cesarean section patients, excluding classical cesarean sections. Obstet Gynecol. 1987;70:713-7.

48. Bofill JA, Vincent RD, Ross EL, Martin RW, Norman PF, Werhan CF, Morrison JC. Nulliparous active labor, epidural analgesia, and cesarean delivery for dystocia. Am J Obstet Gynecol. 1997;177:1465-70.

49. Ramin SM, Gambling DR, Lucas MJ, Sharma SK, Sidawi JE, Leveno KJ. Randomized trial of epidural versus intravenous analgesia during labor. Obstet Gynecol. 1995;86:783-9.

50. Loughnan BA, Carli F, Romney M, Dore CJ, Gordon H. Randomized controlled comparison of epidural bupivacaine versus pethidine for analgesia in labour. Br J Anaesth. 2000;84:715-9.

51. Desprats R, Mandry J, Grandjean H, Amar B, Pontonnier G, Lareng L. Peridural analgesia during labor: comparative study of a fentanyl-marcaine combination and marcaine alone. J Gynecol Obstet Biol Reprod (Paris). 1983;12:901-5.

52. Niv D, Rudick V, Golan A, Chayen MS. Augmentation of bupivacaine analgesia in labor by epidural morphine. Obstet Gynecol. 1986;67:206-9.

53. Phillips GH. Epidural sufentanil/bupivacaine combinations for analgesia during labor: effect of varying sufentanil doses. Anesthesiology. 1987;67:835-8.

54. Vertommen JD, Vandermeulen E, Van Aken H, Vaes L, Soetens M, Van Steenberge A, Mourisse P, Willaert J, Noorduin $\mathrm{H}$, Devlieger H, et al. The effects of the addition of sufentanil to $0.125 \%$ bupivacaine on the quality of analgesia during labor and on the incidence of instrumental deliveries. Anesthesiology. 1991;74:809-14.

55. Yau G, Gregory MA, Gin T, Oh TE. Obstetric epidural analgesia with mixtures of bupivacaine, adrenaline and fentanyl. Anaesthesia. 1990;45:1020-3.

56. Abboud TK, Afrasiabi A, Zhu J, Mantilla M, Reyes A, D'Onofrio L, Khoo N, Mosaad P, Richardson M, Kalra M, et al. Epidural morphine or butorphanol augments bupivacaine analgesia during labor. RegAnesth. 1989;14:115-20.

57. Abboud TK, Zhu J, Afrasiabi A, Reyes A, Sherman G, Khan R, Vera Cruz R, Steffens Z. Epidural butorphanol augments lidocaine sensory anesthesia during labor. Reg Anesth. 1991;16:265-7.

58. Edwards ND, Hartley M, Clyburn P, Harmer M. Epidural pethidine and bupivacaine in labour. Anaesthesia. 1992;47:435-7.

59. Lirzin JD, Jacquinot P, Dailland P, Jorrot JC, Jasson J, Talafre ML, Conseiller C. Controlled trial of extradural bupivacaine with fentanyl, morphine or placebo for pain relief in labour. BrJAnaesth. 1989;62:641-4.

60. Milon D, Lavenac G, Noury D, Allain H, Van den Driessche J, Saint-Marc C. Epidural anesthesia during labor: comparison of 3 combinations of fentanyl-bupivacaine and bupivacaine alone. Ann Fr Anesth Reanim. 1986;5:18-23.

61. Sinatra RS, Goldstein R, Sevarino FB. The clinical effectiveness of epidural bupivacaine, bupivacaine with lidocaine, and bupivacaine with fentanyl for labor analgesia. J Clin Anesth. 1991;3:219-24, discussion 14-5.

62. Viscomi CM, Hood DD, Melone PJ, Eisenach JC. Fetal heart rate variability after epidural fentanyl during labor. Anesth Analg. 1990;71:679-83.

63. Yau G, Gregory MA, Gin T, Bogod DG, Oh TE. The addition of fentanyl to epidural bupivacaine in first stage labour. Anaesth Intensive Care. 1990;18:532-5.

64. Chestnut DH, Owen CL, Bates JN, Ostman LG, Choi WW, Geiger MW. Continuous infusion epidural analgesia during labor: a randomized, double-blind comparison of $0.0625 \%$ bupivacaine $/ 0.0002 \%$ fentanylversus $0.125 \%$ bupivacaine. Anesthesiology. 1988;68:754-9.

65. Elliott RD. Continuous infusion epidural analgesia for obstetrics: bupivacaine versus bupivacaine-fentanyl mixture. Can JAnaesth. 1991;38:303-10.

66. LeeBB, Ngan KeeWD, LauWM, WongAS. Epiduralinfusions for labor analgesia: a comparison of $0.2 \%$ ropivacaine, $0.1 \%$ ropivacaine, and $0.1 \%$ ropivacaine with fentanyl. Reg Anesth Pain Med. 2002;27:31-6.

67. Porter JS, Bonello E, Reynolds F. The effect of epidural opioids on maternal oxygenation during labour and delivery. Anaesthesia. 1996;51:899-903.

68. Rodriguez J, Abboud TK, Reyes A, Payne M, Zhu J, Steffens $Z$, Afrasiabi A. Continuous infusion epidural anesthesia during labor: a randomized, double-blind comparison of $0.0625 \%$ bupivacaine $/ 0.002 \%$ butorphanol and $0.125 \%$ bupivacaine. RegAnesth. 1990;15:300-3.

69. Russell R, Reynolds F. Epidural infusion of low-dose bupivacaine and opioid in labour. Does reducing motor block increase the spontaneous delivery rate? Anaesthesia. 1996;51:266-73.

70. Reynolds F, Russell R, Porter J, Smeeton N. Does the use of lowdosebupivacaine/opioid epiduralinfusionincrease the normal delivery rate? Int J Obstet Anesth. 2003;12:156-63.

71. Camann WR, Denney RA, Holby ED, Datta S. A comparison of intrathecal, epidural, and intravenous sufentanil for labor analgesia. Anesthesiology. 1992;77:884-7.

72. Cesarini M, Torrielli R, Lahaye F, Mene JM, Cabiro C. Sprotte needle for intrathecal anaesthesia for caesarean section: incidence of postdural puncture headache. Anaesthesia. 1990;45:656-8.

73. Devcic A, Sprung J, Patel S, Kettler R, Maitra-D'Cruze A. PDPH in obstetric anesthesia: comparison of 24-gauge Sprotte and 25-gauge Quincke needles and effect of subarachnoid administration of fentanyl. Reg Anesth. 1993;18:222-5.

74. Mayer DC, Quance D, Weeks SK. Headache after spinal anesthesia for cesarean section: a comparison of the 27gaugeQuinckeand24-gaugeSprotteneedles. Anesth Analg. 1992;75:377-80. 
75. Shutt LE, Valentine SJ, Wee MY, Page RJ, Prosser A, Thomas TA. Spinal anaesthesia for caesarean section: comparison of 22-gauge and 25-gauge Whitacre needles with 26-gauge Quincke needles. BrJAnaesth. 1992;69:589-94.

76. Vallejo MC, Mandell GL, Sabo DP, Ramanathan S. Postdural puncture headache: a randomized comparison of five spinal needles in obstetric patients. Anesth Analg. 2000;91:916-20.

77. Hepner DL, Gaiser RR, Cheek TG, Gutsche BB. Comparison of combined spinal-epidural and low dose epidural for labour analgesia. Can J Anaesth. 2000;47:232-6.

78. Kartawiadi L, Vercauteren MP, Van Steenberge AL, Adriaensen HA. Spinal analgesia during labor with low-dose bupivacaine, sufentanil, and epinephrine. A comparison with epidural analgesia. Reg Anesth. 1996;21:191-6.

79. Nickells JS, Vaughan DJ, Lillywhite NK, Loughnan B, Hasan M, Robinson PN. Speed of onset of regional analgesia in labour: a comparison of the epidural and spinal routes. Anaesthesia. 2000;55:17-20.

80. Patel NP, El-Wahab N, Fernando R, Wilson S, Robson SC, Columb MO, Lyons GR. Fetal effects of combined spinalepidural vs epidural labour analgesia: a prospective, randomised double-blind study. Anaesthesia. 2014;69:458-67.

81. Roux M, Wattrisse G, Tai RB, Dufossez F, Krivosic-Horber R. Obstetric analgesia: peridural analgesia versus combined spinal and peridural analgesia. Ann Fr Anesth Reanim. 1999;18:487-98.

82. Sezer OA, Gunaydin B. Efficacy of patient-controlled epidural analgesia after initiation with epidural or combined spinal-epidural analgesia. IntJObstetAnesth. 2007;16:226-30.

83. Vernis L, Duale C, Storme B, Mission JP, Rol B, Schoeffler P. Perispinal analgesia for labour followed by patientcontrolled infusion with bupivacaine and sufentanil: combined spinal-epidural vs. epidural analgesia alone. Eur J Anaesthesiol.2004;21:186-92.

84. Price C, Lafreniere L, Brosnan C, Findley I. Regional analgesia in early active labour: combined spinal epidural vs. epidural. Anaesthesia. 1998;53:951-5.

85. Curry PD, Pacsoo C, Heap DG. Patient-controlled epidural analgesia in obstetric anaesthetic practice. Pain. 1994;57:125-7.

86. Ferrante FM, Barber MJ, Segal M, Hughes NJ, Datta S. $0.0625 \%$ bupivacaine with $0.0002 \%$ fentanyl via patientcontrolled epidural analgesia for pain of labor and delivery. Clin J Pain. 1995;11:121-6.

87. Ferrante FM, Lu L, Jamison SB, Datta S. Patient-controlled epidural analgesia: demand dosing. Anesth Analg. 1991;73:547-52.

88. Gambling DR, Huber CJ, Berkowitz J, Howell P, Swenerton JE, Ross PL, Crochetiere CT, Pavy TJ. Patient-controlled epiduralanalgesiainlabour:varyingbolus doseandlockout interval. Can JAnaesth. 1993;40:211-7.

89. Haydon ML, Larson D, ReedE, Shrivastava VK, Preslicka CW, Nageotte MP.Obstetric outcomes and maternal satisfaction in nulliparous women using patient-controlled epidural analgesia.Am J Obstet Gynecol.2011;205:271.e1-271.e6.

90. Ledin Eriksson S, Gentele C, Olofsson CH. PCEA compared to continuous epidural infusion in an ultra-low-dose regimen for labor pain relief: a randomized study. Acta Anaesthesiol Scand.2003;47:1085-90.

91. Bremerich DH, Waibel HJ, Mierdl S, Meininger D, Byhahn C, Zwissler BC, Ackermann HH. Comparison of continuous background infusion plus demand dose and demand-only parturient-controlled epidural analgesia (PCEA) using ropivacaine combined with sufentanil for labor and delivery. IntJObstetAnesth. 2005;14:114-20.
92. LimY, SiaAT, OcampoCE. Comparison of computerintegrated patient controlled epidural analgesia vs. conventional patient controlled epidural analgesia for pain relief in labour. Anaesthesia. 2006;61:339-44.

93. Missant C, Teunkenst A, Vandermeersch E, Van de Velde M. Patient-controlled epidural analgesia following combined spinal-epidural analgesia in labour: the effects of adding a continuous epidural infusion. Anaesth Intensive Care. 2005;33:452-6.

94. Paech MJ. Patient-controlled epidural analgesia in labourIs a continuous infusion of benefit? Anaesth Intensive Care. 1992;20:15-20.

95. Petry J, Vercauteren M, Van Mol I, Van Houwe P, Adriaensen HA. Epidural PCA with bupivacaine $0.125 \%$, sufentanil 0.75 microgram and epinephrine 1/800.000 for labor analgesia: Is a background infusion beneficial? Acta Anaesthesiol Belg. 2000;51:163-6.

96. Boselli E, Debon R, Cimino Y, Rimmele T, Allaouchiche $B$, Chassard D. Background infusion is not beneficial during labor patient-controlled analgesia with $0.1 \%$ ropivacaine plus $0.5 \mathrm{microg} / \mathrm{ml}$ sufentanil. Anesthesiology. 2004;100:968-72.

97. Wallenborn J.AnalgesieundAnästhesiein der Geburtshilfe. Anästh Intensivmed. 2017;58:66-84.

98. Wong CA, McCarthy RJ, Hewlett B. The effect of manipulation of the programmed intermittent bolus time interval and injection volume on total drug use for labor epidural analgesia: a randomized controlled trial. Anesth Analg. 2011;112:904-11.

99. Dick W, Traub E, Kraus H, Tollner U, Burghard R, Muck J. General anaesthesia versus epidural anaesthesia for primary caesarean section - a comparative study. Eur J Anaesthesiol. 1992;9:15-21.

100. KolatatT,SomboonnanondaA,LertakyamaneeJ,Chinachot T, Tritrakarn T, Muangkasem J. Effects of general and regional anesthesia on the neonate (a prospective, randomized trial). J Med Assoc Thai. 1999;82:40-5.

101. Petropoulos G, Siristatidis C, Salamalekis E, Creatsas G. Spinal and epidural versus general anesthesia for elective cesarean section at term: effect on the acid-base status of the mother and newborn. J Matern Fetal Neonatal Med. 2003;13:260-6.

102. Ryhanen P, Jouppila R, Lanning M, Jouppila P, Hollmen A, Kouvalainen K. Natural killer cell activity after elective cesarean section under general and epidural anesthesia in healthy parturients and their newborns. Gynecol Obstet Invest. 1985;19:139-42.

103. Wallace DH, Leveno KJ, Cunningham FG, Giesecke AH, Shearer VE, Sidawi JE. Randomized comparison of general and regional anesthesia for cesarean delivery in pregnancies complicated by severe preeclampsia. Obstet Gynecol. 1995;86:193-9.

104. Hollmen AI, Jouppila R, Koivisto M, Maatta L, Pihlajaniemi R, Puukka M, Rantakyla P. Neurologic activity of infants following anesthesia for cesarean section. Anesthesiology. 1978;48:350-6.

105. Sener EB, Guldogus F, Karakaya D, Baris S, Kocamanoglu S, Tur A. Comparison of neonatal effects of epidural and general anesthesia for cesarean section. Gynecol Obstet Invest. 2003;55:41-5.

106. Dyer RA, Els I, Farbas J, Torr GJ, Schoeman LK, James MF. Prospective, randomized trial comparing general with spinal anesthesia for cesarean delivery in preeclamptic patients with a nonreassuring fetal heart trace. Anesthesiology. 2003;99:561-9, discussion 5A-6A.

107. KavakZN, Basgul A, Ceyhan N. Short-term outcome of newborn infants: spinal versus general anesthesia for elective 
cesarean section. A prospective randomized study. Eur J Obstet Gynecol Reprod Biol.2001;100:50-4.

108. Mancuso A, De Vivo A, Giacobbe A, Priola V, Maggio Savasta L, Guzzo M, De Vivo D, Mancuso A. General versus spinal anaesthesia for elective caesarean sections: effects on neonatal short-term outcome. A prospective randomised study. J Matern Fetal Neonatal Med. 2010;23:1114-8.

109. Moslemi F, Rasooli S. Comparison of spinal versus general anesthesia for cesarean delivery in patients with severe preeclampsia. J Med Sci. 2007;7(6):1044-8. doi:10.3923/ jms.2007.1044.1048.

110. Shaban M, Ali NS, Abd El-Rezak A. Spinal versus general anesthesia in preeclamptic patients undergoing cesarean delivery. El Minia Med Bull. 2005;16(1):328-43.

111. Lertakyamanee J, Chinachoti T, Tritrakarn T, Muangkasem J, Somboonnanonda A, Kolatat T. Comparison of general and regional anesthesia for cesarean section: success rate, blood loss and satisfaction from a randomized trial. J Med Assoc Thai. 1999;82:672-80.

112. Helbo-Hansen S, Bang U, Garcia RS, Olesen AS, Kjeldsen L. Subarachnoid versus epidural bupivacaine $0.5 \%$ for caesarean section. Acta Anaesthesiol Scand. 1988;32:473-6.

113. McGuinness GA, Merkow AJ, Kennedy RL, Erenberg A. Epidural anesthesia with bupivacaine for cesarean section: neonatal blood levels and neurobehavioral responses. Anesthesiology. 1978;49:270-3.

114. Morgan PJ, Halpern S, Lam-McCulloch J. Comparison of maternal satisfaction between epidural and spinal anesthesia for elective cesarean section. Can J Anaesth. 2000;47:956-61.

115. Olofsson C, Ekblom A, Skoldefors E, Waglund B, Irestedt L. Anesthetic quality during cesarean section following subarachnoid or epidural administration of bupivacaine with or without fentanyl. Acta Anaesthesiol Scand. 1997;41:332-8.

116. Robson SC, Boys RJ, RodeckC, Morgan B. Maternal and fetal haemodynamic effects of spinal and extradural anaesthesia for elective caesarean section. Br JAnaesth. 1992;68:54-9.

117. Sarvela J, Halonen P, Soikkeli A, Korttila K. A double-blinded, randomized comparison of intrathecal and epidural morphine for elective cesarean delivery. Anesth Analg. 2002;95:436-40, table of contents.

118. Schewe JC, Komusin A, Zinserling J, Nadstawek J, Hoeft A, Hering R. Effects of spinal anaesthesia versus epidural anaesthesia for caesarean section on postoperative analgesic consumption and postoperative pain. Eur J Anaesthesiol. 2009;26:52-9.

119. Visalyaputra S, Rodanant O, Somboonviboon W, Tantivitayatan K, Thienthong S, Saengchote W. Spinal versus epidural anesthesia for cesarean delivery in severe preeclampsia: a prospective randomized, multicenter study. Anesth Analg. 2005;101:862-8, table of contents.

120. Berends N, Teunkens A, Vandermeersch E, Van de Velde M. A randomized trial comparing low-dose combined spinalepidural anesthesia and conventional epidural anesthesia for cesarean section in severe preeclampsia. Acta Anaesthesiol Belg. 2005;56:155-62.

121. ChoiDH, KimJA, ChungIS. Comparison of combined spinal epidural anesthesia and epidural anesthesia for cesarean section. Acta Anaesthesiol Scand. 2000;44:214-9.

122. Davies SJ, Paech MJ, Welch H, Evans SF, Pavy TJ. Maternal experience during epidural or combined spinal-epidural anesthesia for cesarean section: a prospective, randomized trial. Anesth Analg. 1997;85:607-13.

123. Karaman S, Akercan F, Akarsu T, Firat V, Ozcan O, Karadadas $\mathrm{N}$. Comparison of the maternal and neonatal effects of epidural block and of combined spinal-epidural block for cesarean section. Eur J Obstet Gynecol Reprod Biol. 2005;121:18-23.

124. Rawal N, Schollin J, Wesstrom G. Epidural versus combined spinal epidural block for cesarean section. Acta Anaesthesiol Scand. 1988;32:61-6.

125. Choi DH, Ahn HJ, Kim JA. Combined low-dose spinalepidural anesthesia versus single-shot spinal anesthesia for elective cesarean delivery. IntJObstetAnesth. 2006;15:13-7.

126. Choi DH, Park NK, Cho HS, Hahm TS, Chung IS. Effects of epidural injection on spinal block during combined spinal and epidural anesthesia for cesarean delivery. Reg Anesth Pain Med. 2000;25:591-5.

127. Salman C, Kayacan N, Ertugrul F, BigatZ, Karsli B. Combined spinal-epidural anesthesia with epidural volume extension causes a higher level of block than single-shot spinal anesthesia. BrazJ Anesthesiol. 2013;63:267-72.

128. Thoren T, Holmstrom B, Rawal N, Schollin J, Lindeberg S, Skeppner G. Sequential combined spinal epidural block versus spinal blockfor cesarean section: effects on maternal hypotension and neurobehavioral function of the newborn. Anesth Analg. 1994;78:1087-92.

129. Husaini SW, Russell IF. Volume preload: lack of effect in the prevention of spinal-induced hypotension at caesarean section. Int JObstetAnesth. 1998;7:76-81.

130. Kamenik M, Paver-Erzen V. The effects of lactated Ringer's solution infusion on cardiac output changes after spinal anesthesia. Anesth Analg. 2001;92:710-4.

131. Mojica JL, Melendez HJ, Bautista LE. The timing of intravenous crystalloid administration and incidence of cardiovascular side effects during spinal anesthesia: the results from a randomized controlled trial. Anesth Analg. 2002;94:432-7, table of contents.

132. Ngan KeeWD, Khaw KS, Lee BB, NgFF, Wong MM. Randomized controlled study of colloid preload before spinal anaesthesia for caesarean section. BrJAnaesth. 2001;87:772-4.

133. Kee NWD, Khaw KS, Lee BB, Wong MM, Ng FF. Metaraminol infusion for maintenance of arterial blood pressure during spinal anesthesia for cesarean delivery: the effect of a crystalloid bolus. Anesth Analg. 2001;93:703-8.

134. Nishikawa K, Yokoyama N, Saito S, Goto F. Comparison of effects of rapid colloid loading before and after spinal anesthesia on maternal hemodynamics and neonatal outcomes in cesarean section. J Clin Monit Comput. 2007;21:125-9.

135. Lee SY, Choi DH, Park HW. The effect of colloid cohydration on the use of phenylephrine and hemodynamics during low-dose combined spinal-epidural anesthesia for cesarean delivery. Korean J Anesthesiol. 2008;55(6):685. doi:10. 4097/kjae.2008.55.6.685.

136. Desalu I, Kushimo OT. Is ephedrine infusion more effective at preventing hypotension than traditional prehydration during spinal anaesthesia for caesarean section in African parturients? Int J Obstet Anesth. 2005;14:294-9.

137. King SW, Rosen MA. Prophylactic ephedrine and hypotension associated with spinal anesthesia for cesarean delivery. IntJObstet Anesth. 1998;7:18-22.

138. Loughrey JP, Walsh F, Gardiner J. Prophylactic intravenous bolus ephedrine for elective caesarean section under spinal anaesthesia. Eur JAnaesthesiol. 2002;19:63-8.

139. Ngan Kee WD, Khaw KS, Lee BB, Lau TK, Gin T. A doseresponse study of prophylactic intravenous ephedrine for the prevention of hypotension during spinal anesthesia for cesarean delivery. Anesth Analg. 2000;90:1390-5.

140. Ramin SM, Ramin KD, Cox K, Magness RR, Shearer VE, Gant NF. Comparison of prophylactic angiotensin IIversus ephedrine infusion for prevention of maternal hypotension during spinal anesthesia. AmJ Obstet Gynecol. 1994;171:734-9. 
141. Ayorinde BT, Buczkowski P, Brown J, Shah J, Buggy DJ. Evaluation of pre-emptive intramuscular phenylephrine and ephedrine for reduction of spinal anaesthesia-induced hypotension during caesarean section. Br J Anaesth. 2001;86:372-6.

142. Gutsche BB. Prophylactic ephedrine preceding spinal analgesia for cesarean section. Anesthesiology. 1976;45:462-5.

143. WebbAA, ShiptonEA. Re-evaluation ofim ephedrineas prophylaxis against hypotension associated with spinal anaesthesia for Caesarean section. Can JAnaesth. 1998;45:367-9.

144. Allen TK, George RB, White WD, Muir HA, Habib AS. A double-blind, placebo-controlled trial of four fixed rate infusion regimens of phenylephrinefor hemodynamic support during spinal anesthesia for cesarean delivery. Anesth Analg. 2010;111:1221-9.

145. Langesaeter E, Rosseland LA, StubhaugA. Continuousinvasive blood pressure and cardiac output monitoring during cesarean delivery: arandomized, double-blind comparison of low-dose versus high-dose spinal anesthesia with intravenous phenylephrine or placeboinfusion. Anesthesiology. 2008;109:856-63.

146. Siddik-Sayyid SM, Taha SK, Kanazi GE, Aouad MT. A randomized controlled trial of variabe rate phenylephrine infusion with rescue phenylephrine boluses versus rescue boluses alone on physician interventions during spinal anesthesia for elective cesarean delivery. Anesth Analg. 2014;118(3):611-8. doi:10.1213/01.ane.0000437731.60260. ce.

147. Alahuhta S, Rasanen J, Jouppila P, Jouppila R, Hollmen AI. Ephedrine and phenylephrine for avoiding maternal hypotension due to spinal anaesthesia for caesarean section. Effects on uteroplacental and fetal haemodynamics. Int J ObstetAnesth. 1992;1:129-34.

148. Cooper DW, Carpenter M, Mowbray P, Desira WR, Ryall DM, Kokri MS. Fetal and maternal effects of phenylephrine and ephedrine during spinal anesthesia for cesarean delivery. Anesthesiology. 2002;97:1582-90.

149. Cooper DW, Jeyaraj L, Hynd R, Thompson R, Meek T, Ryall DM, Kokri MS. Evidence that intravenous vasopressors can affect rostral spread of spinal anesthesia in pregnancy. Anesthesiology. 2004;101:28-33.

150. Hall PA, Bennett A, Wilkes MP, Lewis M. Spinal anaesthesia for caesarean section: comparison of infusions of phenylephrine and ephedrine. BrJAnaesth. 1994;73:471-4.

151. Ngan Kee WD, Khaw KS, Tan PE, Ng FF, Karmakar MK. Placental transfer and fetal metabolic effects of phenylephrine and ephedrine during spinal anesthesia for cesarean delivery. Anesthesiology. 2009;111:506-12.

152. Ngan KeeWD, LeeA, Khaw KS, NgFF, Karmakar MK, Gin T. A randomized double-blinded comparison of phenylephrine and ephedrine infusion combinations to maintain blood pressure during spinal anesthesia for cesarean delivery: the effects on fetal acid-base status and hemodynamic control. Anesth Analg. 2008;107:1295-302.

153. Daley MD, Sandler AN, Turner KE, Vosu H, Slavchenko P. A comparison of epidural and intramuscular morphine in patients following cesarean section. Anesthesiology. 1990;72:289-94.

154. Eisenach JC, Grice SC, Dewan DM. Patient-controlled analgesia following cesarean section: a comparison with epidural and intramuscular narcotics. Anesthesiology. 1988;68:444-8.

155. Harrison DM, Sinatra R, Morgese L, Chung JH. Epidural narcoticand patient-controlledanalgesiaforpost-cesarean section pain relief. Anesthesiology. 1988;68:454-7.

156. Henderson SK, Matthew EB, Cohen H, Avram MJ. Epidural hydromorphone: a double-blind comparison with intramuscular hydromorphone for postcesarean section analgesia. Anesthesiology. 1987;66:825-30.

157. Macrae DJ, Munishankrappa S, Burrow LM, Milne MK, Grant IS. Double-blind comparison of the efficacy of extradural diamorphine, extradural phenoperidine and i.m. diamorphine following caesarean section. Br J Anaesth. 1987;59:354-9.

158. Perriss BW, Latham BV, Wilson IH. Analgesia following extradural and i.m. pethidine in post-caesarean section patients. BrJAnaesth. 1990;64:355-7.

159. Smith ID, Klubien KE, Wood ML, Macrae DJ, Carli F. Diamorphine analgesia after caesarean section. Comparison of intramuscular and epidural administration of four dose regimens. Anaesthesia. 1991;46:970-3.

160. Chambers WA, Mowbray A, Wilson J. Extradural morphine for the relief of pain following caesarean section. Br J Anaesth. 1983;55:1201-3.

161. Cohen S, Pantuck CB, Amar D, Burley E, Pantuck EJ. The primary action of epidural fentanyl after cesarean delivery is via a spinal mechanism. Anesth Analg. 2002;94:674-9, table of contents.

162. Cohen SE, Tan S, White PF. Sufentanil analgesia following cesarean section: epidural versus intravenous administration. Anesthesiology. 1988;68:129-34.

163. Parker RK, Sawaki Y, White PF. Epidural patient-controlled analgesia: influence of bupivacaine and hydromorphone basal infusion on pain control after cesarean delivery. Anesth Analg. 1992;75:740-6.

164. Rosen MA, Hughes SC, Shnider SM, Abboud TK, Norton M, Dailey PA, Curtis JD. Epidural morphine for the relief of postoperative pain after cesarean delivery. Anesth Analg. 1983;62:666-72.

165. Alfirevic Z, Elbourne D, Pavord S, Bolte A, Van Geijn H, Mercier F, Ahonen J, Bremme K, Bodker B, Magnusdottir EM, Salvesen K, Prendiville W, Truesdale A, Clemens F, Piercy D, Gyte G. Use of recombinant activated factor VII in primary postpartum hemorrhage: the Northern European registry 2000-2004. Obstet Gynecol.2007;110:1270-8.

166. King M, Wrench I, Galimberti A, Spray R. Introduction of cell salvage to a large obstetric unit: the first six months. Int J Obstet Anesth. 2009;18:111-7.

167. Kjaer K, Comerford M, Gadalla F. General anesthesia for cesarean delivery in a patient with paroxysmal nocturnal hemoglobinuria and thrombocytopenia. Anesth Analg. 2004;98:1471-2, table of contents.

168. Lilker SJ, Meyer RA, Downey KN, MacArthur AJ. Anesthetic considerations for placenta accreta. Int J Obstet Anesth. 2011;20:288-92.

169. Margarson MP. Delayed amniotic fluid embolism following caesarean section under spinal anaesthesia. Anaesthesia. 1995;50:804-6.

170. Nagy CJ, Wheeler AS, Archer TL. Acute normovolemic hemodilution, intraoperative cell salvage and PulseCO hemodynamic monitoring in a Jehovah's Witness with placenta percreta. Int JObstetAnesth. 2008;17:159-63.

171. Potter PS, Waters JH, Burger GA, Mraovic B. Application of cell-salvage during cesarean section. Anesthesiology. 1999;90:619-21.

172. Rogers WK, Wernimont SA, Kumar GC, Bennett E, Chestnut DH. Acute hypotension associated with intraoperative cell salvage using a leukocyte depletion filter during management of obstetric hemorrhage due to amniotic fluid embolism. Anesth Analg. 2013;117:449-52.

173. Ferouz F, Norris MC, Leighton BL. Risk of respiratory arrest afterintrathecal sufentanil. Anesth Analg. 1997;85:1088-90.

174. Godley M, Reddy AR. Use of LMA for awake intubation for caesarean section. Can J Anaesth. 1996;43:299-302. 
175. Greenhalgh CA. Respiratory arrest in a parturient following intrathecal injection of sufentanil and bupivacaine. Anaesthesia. 1996;51:173-5.

176. Hawksworth CR, Purdie J. Failed combined spinal epidural thenfailedintubation at an elective caesarean section. Hosp Med. 1998;59:173.

177. Hinchliffe D, Norris A. Management of failed intubation in a septic parturient. BrJAnaesth. 2002;89:328-30.

178. Kehl F, Erfkamp S, Roewer N. Respiratory arrest during caesarean section after intrathecal administration of sufentanil in combination with $0.1 \%$ bupivacaine $10 \mathrm{ml}$. Anaesth Intensive Care. 2002;30:698-9.

179. Keller C, Brimacombe J, Lirk P, Puhringer F. Failed obstetric tracheal intubation and postoperative respiratory support with the ProSeal laryngeal mask airway. Anesth Analg. 2004;98:1467-70.

180. Parker J, Balis N, Chester S, Adey D. Cardiopulmonary arrest in pregnancy: successful resuscitation of mother and infant followingimmediate caesarean section in labour ward. Aust NZJObstet Gynaecol. 1996;36:207-10.

181. PopatMT, ChippaJH, Russell R. Awakefibreopticintubation following failed regional anaesthesia for caesarean section in a parturient with Still's disease. Eur J Anaesthesiol. 2000;17:211-4.

182. Neuhaus S, Neuhaus C, Fluhr H, Hofer S, Schreckenberger R, Weigand MA, Bremerich D. „Why mothers die“. Learning from the analysis of anaesthesia-related maternal deaths (1985-2013). Anaesthesist. 2016;65:281-94.
183. Mushambi MC, Kinsella SM, Popat M, Swales H, Ramaswamy KK, Winton AL, Quinn AC. Obstetric Anaesthetists' Association and Difficult Airway Society guidelines for the management of difficult and failed tracheal intubation in obstetrics. Anaesthesia. 2015;70:1286-306.

184. ÖGARI ApGd. Regionalanästhesie unter gerinnungshemmender Medikation. 2016

185. Fachgesellschaften AdWM. S1 - Leitlinie: Hygieneempfehlungen für die Regionalanästhesie. 2014.

186. Fachgesellschaften AdWM. S2k - Leitlinie: Peripartale Blutungen, Diagnostikund Therapie. 2016.

187. Fachgesellschaften AdWM. S1 - Leitlinie: Hypertensive Schwangerschaftserkrankungen:DiagnostikundTherapie. 2013.

188. Fachgesellschaften AdWM. S1 - Leitlinie: Anwendung des CTG während Schwangerschaft und Geburt. 2013.

189. Fachgesellschaften AdWM. S2k - Leitlinie: Frühgeborene an der Grenze der Lebensfähigkeit. 2014.

190. Deutsche Gesellschaft für Gynäkologie und Geburtshilfe, Arbeitsgemeinschaft Medizinrecht, Deutsche Gesellschaft für Anästhesiologie und Intensivmedizin, Berufsverband Deutscher Anästhesisten. Zur Frage der postoperativen Überwachung von Kaiserschnittpatientinnen. Anästh Intensivmed. 2016;57:47-50.

191. Intensivmedizin DGfAu. Einsatz von Lachgas zur Schmerztherapie unter der Geburt. Anästh Intensivmed. 2014;55: 679-82. 